\begin{tabular}{c} 
International Journal of Scientific World, $4(2)(2016) 52-56$ \\
International Journal of Scientific World \\
SPC \\
Website: $\begin{array}{c}\text { www. sciencepubco.com/index. } h \text { h } / I J S W \\
\text { doi: } 10.14419 / \text { ijsw.v4i2.6474 } \\
\text { Research paper }\end{array}$ \\
\hline
\end{tabular}

\title{
Numerical behavior of a fractional order dynamical model of RNA silencing
}

\author{
A.M.A. El-Sayed ${ }^{1}$, M. Khalil ${ }^{2}$ *, A.A.M. Arafa ${ }^{3}$, Amaal Sayed ${ }^{3}$ \\ ${ }^{1}$ Department of mathematics, Faculty of Science, Alexandria University, Alexandria, Egypt \\ ${ }^{2}$ Department of mathematics, Faculty of Engineering, October university for modern sciences and arts (MSA), Giza, Egypt \\ ${ }^{3}$ Department of mathematics, Faculty of Science, Port Said University, Port Said, Egypt \\ *Corresponding authorE-mail: mkibrahim@msa.eun.eg
}

\begin{abstract}
A class of fractional-order differential models of RNA silencing with memory is presented in this paper. We also carry out a detailed analysis on the stability of equilibrium and we show that the model established in this paper possesses non-negative solutions. Numerical solutions are obtained using a predictor-corrector method to handle the fractional derivatives. The fractional derivatives are described in the Caputo sense. Numerical simulations are presented to illustrate the results. Also, the numerical simulations show that, modeling the phenomena of RNA silencing by fractional ordinary differential equations (FODE) has more advantages than classical integer-order modeling.
\end{abstract}

Keywords: Fractional Calculus; RNA Silencing Fractional Order Model; Predictor-Corrector Method.

\section{Introduction}

DNA and RNA perform various functions in humans. RNA is vital to cells because it broadcast information encoded in DNA to tiny organs within the cell [34]. There are different kinds of RNAs like microRNAs (miRNAs), the so called messenger RNAs (mRNAs), the double-stranded RNA (dsRNA) which is RNA with two complementary strands, and small interfering RNA (siRNA) which is a class of double-stranded RNA [8]. Cells use miRNAs to control the number of protein molecules made from mRNAs [33]. In the last few decades, RNA silencing has become a major focus of genome sciences around the world [14]. RNA silencing (also known as RNA interference) is a sequence-specific RNA degradation mechanism that occurs in a broad range of eukaryotic organisms [25]. It is based on an immune system that protects eukaryotes against viruses [7]. RNA silencing also plays a primary antiviral role in plants and in insects [30]. So, RNA silencingbased resistance has been an impressive tool that has been used to engineer resistant crops [25]. Mathematical modeling has become an essential tool to understand the dynamics of RNA silencing [14], [30]. Different models have been presented to describe the dynamics of RNA silencing, but these models have been restricted to integer order (delay) differential equations [22], [26]. Hence, we propose in this paper a system of FODE for modeling RNA silencing based on the integer order model in [8]. The major reason of using is that FODE are naturally related to systems with memory which exists in most biological systems [3], [4], [9], [15], [17]. Also, they are closely related to fractals, which are abundant in biological systems [13], [16], [19], [20], [21]. FODE are, at least, as stable as their integer order counterpart [1], [2], [10], [11], [23], [24]. The rest of the paper is organized as follows. A brief review of the fractional calculus theory is given in Section 2. A discussion about the equilibrium points and stability is presented in section 3 while in section 4 , we discuss the existence and uniqueness of the presented fractional order model. Section 5 is devoted for the numerical solution of the presented model.

\section{Model derivation}

First of all, some definitions of fractional order integrals and derivatives [5], [6] are presented here. For the concept of fractional derivative, we will adopt Caputo's definition, which is a modification of the Riemann-Liouville definition and has the advantage of dealing properly with initial value problems.

Definition 2.1: The fractional integral of order $\alpha>0$ of a function $f: \mathfrak{R}^{+} \rightarrow \mathfrak{R}$ is given by

$$
\begin{aligned}
& J^{(\alpha)} f(x)=\frac{1}{\Gamma(\alpha)} \int_{0}^{x}(x-t)^{\alpha-1} f(t) d t, \quad \alpha>0, \quad x>0 \\
& J^{0} f(x)=f(x)
\end{aligned}
$$

Definition 2.2: Riemann-Liouville and Caputo fractional order derivatives of a continuous function $f: \mathfrak{R}^{+} \rightarrow \mathfrak{R}$ is given respectively by

$$
\begin{aligned}
& D^{(\alpha)} f(x)=D^{m}\left(J^{m-\alpha} f(x)\right), \\
& D_{*}^{(\alpha)} f(x)=J^{m-\alpha}\left(D^{m} f(x)\right)
\end{aligned}
$$

Where

$$
m-1<\alpha \leq m, \quad m \in N
$$


The definition of fractional derivative involves an integration which is non-local operator (as it is defined on an interval) so fractional derivative is a non-local operator [27], [29], [31], [32]. In other words, calculating time-fractional derivative of a function $f(t)$ at some time $t=t_{1}$ requires all the previous history, i.e. all $f(t)$ from $t=0$ to $t=t_{1}$.

Now we introduce fractional-order into the model of RNA silencing [8]. The new system is described by the following set of FODE:

$$
\begin{aligned}
& D^{(\alpha)}(S)=-a S+g C, \\
& D^{(\alpha)}(R)=a n S-d_{R} R-b R M, \\
& D^{(\alpha)}(C)=b R M-\left(g+d_{C}\right) C, \\
& D^{(\alpha)}(M)=h-d_{M} M-b R M .
\end{aligned}
$$

Where $0<\alpha \leq 1, S(t), R(t), C(t)$ and $M(t)$ present the concentrations of the dsRNA، RISC, RISC-mRNA complex, and mRNA at time $t$, respectively. The parameters can be defined as follows: $a$ is the rate of dsRNA degradation by Dicer.

$b$ is mass action rate constant for RISC-mRNA formation.

$h$ is the rate of target mRNA synthesis.

$g$ is the rate of dsRNA synthesis from RISC-mRNA complex.

$d_{M}$ is the rate of nonspecific mRNA degradation.

$d_{R}$ is the rate of RISC dissociation.

$d_{C}$ is the Rate at which complex is destroyed.

$n$ is the Number of siRNAs produced from one secondary dsRNA.

The initial conditions are $S(O)=S_{0}, R(O)=R_{0}, C(O)=C_{0}$, and $M(O)=M_{0}$ the basic reproductive number $R_{0}$ for dsRNA is presented in $[8]$ as

$$
R_{o}=\frac{n g b h}{\left(g+d_{C}\right)\left(b h+d_{R} d_{M}\right)} .
$$

If $R_{0}<1$, then the silencing reaction will take off.

\section{Equilibrium points and stability}

The authors in [8] deduced the equilibrium Points of the integer order system of the given model (3), i.e. when $\alpha=1$ in (3). To evaluate the equilibrium points of the fractional-order system (3), let

$$
D^{(\alpha)}(S)=0, D^{(\alpha)}(R)=0, D^{(\alpha)}(C)=0, D^{(\alpha)}(M)=0 .
$$

Model (1) has two steady states: $E_{0}=\left(0,0,0, \frac{h}{d_{M}}\right)$

and $E_{l}=\left(S^{*}, R^{*}, C^{*}, M^{*}\right)$ where:

$$
\begin{aligned}
S^{*} & =\frac{g}{a} C^{*}, \\
R^{*} & =\frac{\beta C^{*}}{d_{R}}, \\
C^{*} & =\frac{\beta h-d_{R} d_{M}\left(g+d_{C}\right)}{\beta\left(d_{C}+g\right)}, \\
M^{*} & =\frac{\left(d_{C}+g\right) d_{R}}{\beta b} .
\end{aligned}
$$

Where $\beta=g(n-1)-d_{C}$.
The biological meaning of steady state $E_{0}$ is that, the silencing does not occur. The second steady state $E_{l}$ is biologically meaningful only if $S^{*}, R^{*}, C^{*}$, and $M^{*}$ only are nonnegative [8], for which the condition $\beta h>\left(d_{R} d_{M} / b\right)\left(d_{C}+g\right)$ is both necessary and sufficient.

Also, a sufficient condition for the local asymptotic stability of the equilibrium points is that the eigenvalues $\lambda_{i}$ of the Jacobian matrix of $E_{1}$ satisfy the condition $\left|\arg \left(\lambda_{i}\right)\right|>\alpha \frac{\pi}{2}$ [2], [11], [23]. This confirms that fractional-order differential equations are, at least, as stable as their integer order counterpart.

\section{Existence of uniformly stable solution}

To prove the existence and uniqueness of solution for the system (3). Firstly we will recall the following lemma:

Lemma 4.1: (Theorem 8.11, [12]) Let $0<\alpha_{j}<1$, for $j=1,2, \ldots, q$ and consider the initial value problem given by the multi-order fractional differential system (in Caputo sense)

$D^{\alpha} y_{j}=f_{j}\left(x, y_{1}(x), y_{2}(x), \ldots, y_{q}(x)\right), \quad j=1,2, \ldots, q$

With initial condition

$$
y_{j}(O)=p_{j}, \quad j=1,2, \ldots, q \text {. }
$$

Assume that the functions $f_{j}=[0, x] \times R^{q} \rightarrow R, j=1,2, \ldots, q$ are continuous and satisfy Lipschitz conditions with respect to all their arguments except for the first. Then the initial value problem (4) has a uniquely determined continuous solution. Since each $f_{i}=\left[0, T_{1}\right] \times R^{4} \rightarrow R_{+} ; i=1,2,3,4$ is continuous.

To prove that the system (3) has a unique continuous solution, we want to show that each $f_{i}$ satisfies the Lipschitz condition with respect to each of its argument except for the first. Let

$$
\begin{aligned}
& x_{I}(t)=S(t), x_{2}(t)=R(t), x_{3}(t)=C(t), x_{4}(t)=M(t) \\
& D^{\alpha} x_{1}(t)=f_{I}\left(x_{1}(t), x_{2}(t), x_{3}(t), x_{4}(t)\right), t>0 \quad \text { and } x_{1}(0)=x_{01} \text {, } \\
& D^{\alpha} x_{2}(t)=f_{2}\left(x_{1}(t), x_{2}(t), x_{3}(t), x_{4}(t)\right), t>0 \text { and } x_{2}(0)=x_{02},(6) \\
& D^{\alpha} x_{3}(t)=f_{3}\left(x_{1}(t), x_{2}(t), x_{3}(t), x_{4}(t)\right), t>0 \quad \text { and } x_{3}(0)=x_{03} \text {, } \\
& D^{\alpha} x_{4}(t)=f_{4}\left(x_{1}(t), x_{2}(t), x_{3}(t), x_{4}(t)\right), t>0 \quad \text { and } x_{4}(0)=x_{04} \text {, }
\end{aligned}
$$

Let $D=\left\{x_{1}, x_{2}, x_{3}, x_{4} \in R:\left|x_{i}(t) \leq r\right|, t \in[0, T], i=1,2,3,4\right\}$,

The function $f_{i}$ satisfy the Lipschitz condition on $R_{+}^{4}$ if

$\left|f_{i}\left(S_{1}, R_{1}, C_{1}, M_{1}\right)-f_{i}\left(S_{2}, R_{2}, C_{2}, M_{2}\right)\right| \leq \eta\left(\left|S_{1}-S_{2}\right|+\right.$

$\left|R_{1}-R_{2}\right|+\left|C_{1}-C_{2}\right|+\left|M_{1}-M_{2}\right|$

Where $\eta$ is the Lipschitz constant. Then on D we have $\left|\frac{\partial}{\partial x_{j}} f_{i}\left(x_{1}, x_{2}, x_{3}, x_{4}\right)\right| \leq \eta_{n}, \forall i, j=1,2,3,4$, and $n=1,2,3, \ldots 16$ 
This implies that each of the four functions $f_{1}, f_{2}, f_{3}, f_{4}$ satisfies the Lipschitz condition with respect to the four arguments $x_{1}, x_{2}, x_{3}, x_{4}$ and then each of the four functions $f_{1}, f_{2}, f_{3}, f_{4}$ is absolutely continuous with respect to the four arguments $x_{1}, x_{2}, x_{3}, x_{4}$.

Consider the following initial value problem which represents the fractional-order RNA silencing (3)

Definition 4.1: By a solution of the fractional-order RNA silencing (5), (6), (7), and (8) which is a column vector

$X(T)=\left(x_{1}(t), x_{2}(t), x_{3}(t), x_{4}(t)\right)^{t}, x_{1}, x_{2}, x_{3}, x_{4}$

And $x_{4} \in C[0, T], T<\infty$ where $C[0, T]$ the class of continuous functions defined on the interval $[0, T]$ and $\tau$ denotes the transpose of the matrix [18].

Theorem 4.1: The fractional order model which describes a vector-borne plant disease model (5), (6), (7), and (8) has a unique uniformly Lyapunov stable solution [18].

Proof: Write the model 5, 6, 7 and 8 in the matrix form

$D^{\alpha} X(t)=F(X(t)), t>0$ and $X(0)=x_{0}$ where

$X(t)=\left(x_{1}(t), x_{2}(t), x_{3}(t), x_{4}(t)\right)^{\tau}, F(X(t))=\left(f_{1}, f_{2}, f_{3}, f_{4}\right)^{\tau}$

By applying Theorem 2.1 [18], we deduce that the fractional order RNA silencing system (5), (6), (7), and (8) has a unique solution. Also by Theorem 3.2 [18] this solution is uniformly Lyapunov stable.

\section{Numerical simulation and discussion}

In this section, predictor-corrector method is applied to get numerical solutions of the system (1). The values of the parameters are considered as in [8] as follows:

$a=10, b=0.001, h=1000, g=1, d_{M}=1, d_{R}=0.1, d_{C}=1, n=5$.

The initial conditions are:

$M(0)=1000, R(0)=0, C(0)=0, S(0)=10$ (Fig. 1 and Fig. 2) or $\mathrm{S}(0)=1000$ (Fig. 3).

By using these values of parameters, it is clear that $R_{0}>1$. It means that a small amount of dsRNA will be sufficient to trigger a silencing reaction in this case.

(A)

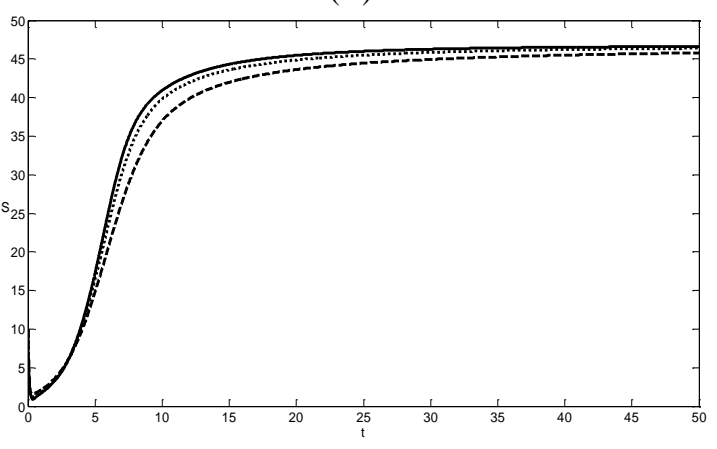

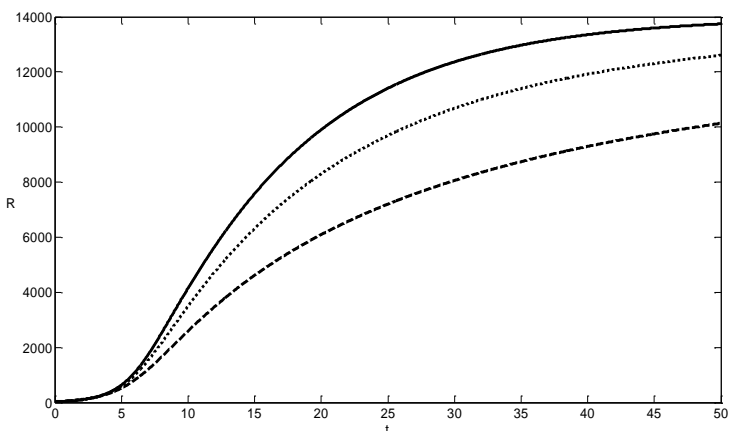

Fig. 1: A) the Concentration of the $S(t)$, B) the Concentration of the $R(t)$ for $\alpha=1$ (the Solid Line) $\alpha=0.9$ (the Dotted Line), $\alpha=0.75$ (The Dashed Line) in the 1st Case: $\mathrm{t}=$ Time, $\mathrm{S}=$ Concentration of dsRNA, R=Concentration of RISC.

(A)

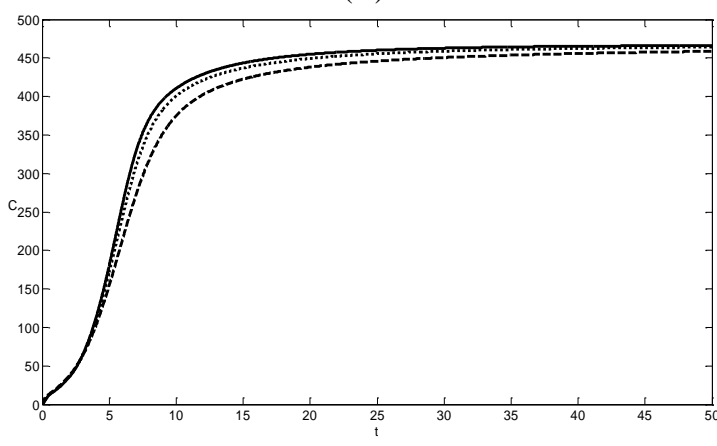

(B)

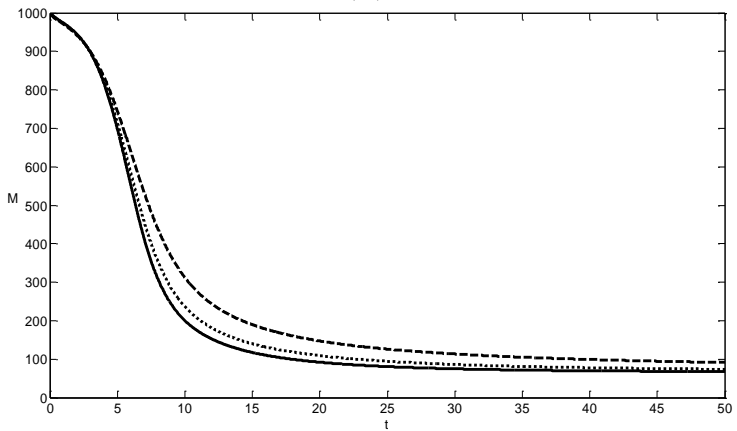

Fig. 2: A) the Concentration of the $C(t)$, B) the Concentration of $M(t)$ for $\alpha=1$ (the Solid Line) $\alpha=0.9$ (the Dotted Line), $\alpha=0.75$ (the Dashed Line) in the $1^{\text {st }}$ Case, $\mathrm{t}=$ Time, $\mathrm{C}=$ Concentration of RISC-mRNA, $\mathrm{M}=$ Concentration of mRNA.

(A) 
(B)

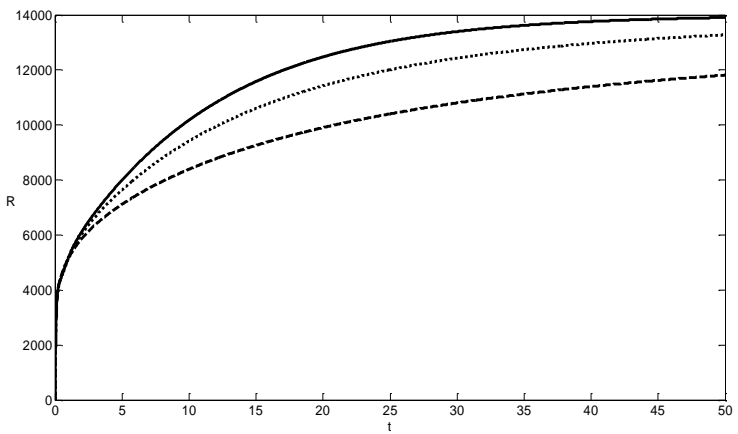

(C)

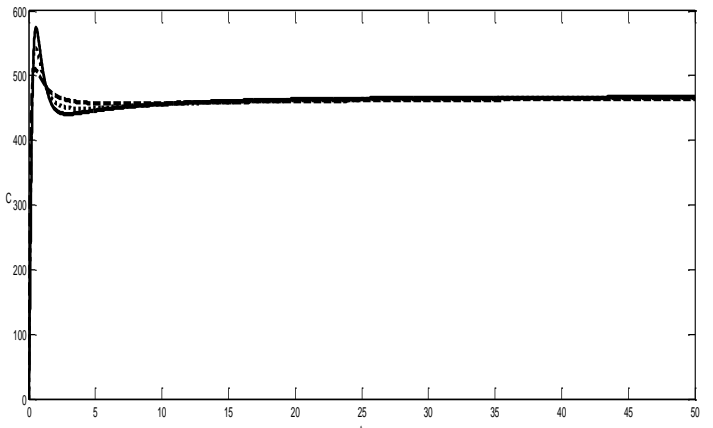

(D)

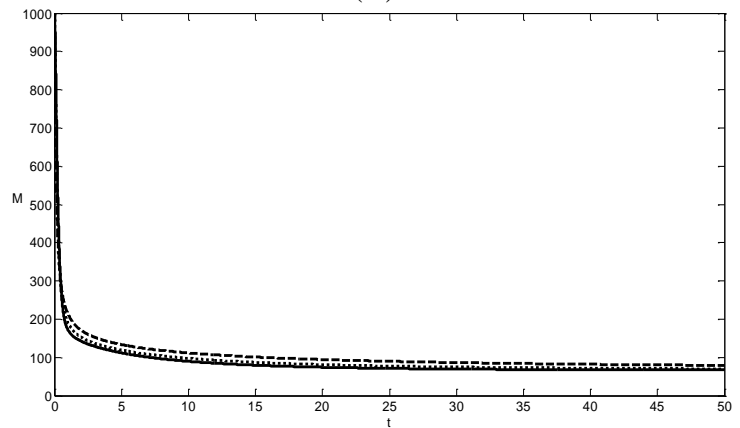

Fig. 3: (A) the Concentrations of the $S(t)$, (B) the Concentrations of the $R(t)$, (C) the Concentrations of the $C(t)$, (D) the Concentrations Of the $M(t)$ for $\alpha=1$ (the Solid Line) $\alpha=0.9$ (the Dotted Line), $\alpha=0.75$ (The Dashed Line) in the $2^{\text {nd }}$ Case, $\mathrm{t}=$ Time, $\mathrm{S}=$ Concentration of dsRNA, R=Concentration of RISC, C=Concentration of RISC-mRNA, $\mathrm{M}=$ Concentration of mRNA.

\section{Conclusion}

In this paper, the numerical solution of fractional order model of RNA silencing is discussed. We show that, fractional-order differential equations are generalizations of integer-order differential equations. In Fig. 1, Fig. 2 and Fig.3, the same degree of silencing is obtained for both low and high initial dsRNA concentrations. When $\alpha \rightarrow 1$ the solution of the fractional model (3) $S_{\alpha}(t), R_{\alpha}(t), C_{\alpha}(t), M_{\alpha}(t)$ reduce to the standard solution $S(t), R(t), C(t), M(t)$, (see Fig.1, Fig.2 and Fig.3). In addition of proving the existence and uniqueness of a stable solution. Also the results show that the numerical simulations confirm the advantages of the numerical technique and using fractional-order differential models in biological systems over the differential equations with integer order.

\section{Acknowledgements}

The authors are grateful to the referees for their useful comments and valuable recommendations.

\section{References}

[1] E. Ahmed and A.S. Elgazzar," On fractional order differential equations model for nonlocal epidemics", PHYSICA A, 379 (2007) 607-614. http://dx.doi.org/10.1016/i.physa.2007.01.010.

[2] E. Ahmed, A.M.A. El-Sayed, H.A.A. El-Saka," Equilibrium points, stability and numerical solutions of fractional-order predator-prey and rabies models", J. Math. Anal. Appl. 325 (2007) 542-553, http://dx.doi.org/10.1016/j.jmaa.2006.01.087.

[3] F. Aini Abdullah and Ahmad Izani Md. Ismail, Simulations of the Spread of the Hantavirus Using Fractional Differential Equations, Matematika, 27(2011) 149-158.

[4] A.A.M. Arafa, S.Z. Rida, M. Khalil," Fractional modeling dynamics of HIV and CD4+ T-cells during primary infection", Nonlinear Biomedical Physics 6(2012) 1-7, http://dx.doi.org/10.1186/17534631-6-1.

[5] A.A.M. Arafa, S.Z. Rida, M. Khalil," The effect of anti-viral drug treatment of human immunodeficiency", Appl. Math. Model, 37 (2013) 2189-2196,http://dx. doi:10.1016/j.apm.2012.05.002 http://dx.doi.org/10.1016/j.apm.2012.05.002.

[6] A.A.M. Arafa, S.Z. Rida. M. Khalil," Solutions of Fractional model of human T-cell lymphotropic virus I (HTLV-I) infection of CD4+ T-cells using HAM", International Journal of Basic and Applied $\begin{array}{lllll}\text { Sciences, } & 1 & \text { (1) } & \text { (2012) }\end{array}$ http://dx.doi.org/10.14419/ijbas.v1i1.15.

[7] D. Baulcombe, "RNA silencing", Curr. Biol. 12, (2002) R82-R84, http://dx.doi.org/10.1016/S0960-9822(02)00665-6..

[8] C.T. Bergstrom, E. McKittrick, R. Antia," Mathematical models of RNA silencing: Unidirectional amplification limits accidental selfdirected reactions", PNAS, 100 (2003) 11511-11516. http://dx.doi.org/10.1073/pnas.1931639100.

[9] M. Dalir, M. Bashour," Applications of Fractional Calculus", Appl. Math. Sci. 4 (2010) 1021-1032.

[10] E. Demirci, Arzu Unal and Nuri Ozalp," A fractional order SEIR Model with density dependent death rate", Hacet. J. Math. Stat., 40 (2) (2011) $287-295$

[11] W. Deng," Smoothness and stability of the solutions for nonlinear fractional differential equations", Nonlinear Anal-Theor. 72 (2010) 1768-1777, http://dx.doi.org/10.1016/j.na.2009.09.018.

[12] K. Diethelm," The Analysis of Fractional Differential Equations", Springer-Verlag, Berlin, (2010) 181-182.

[13] Y. Ding, H. Yea," A fractional-order differential equation model of HIV infection of CD4+T-cells", Math. Comput. Model. 50 (2009) 386-392, http://dx.doi.org/10.1186/1753-4631-6-1.

[14] A. Eamens, M.B. Wang, N. A. Smith, P. M. Waterhouse," RNA Silencing in Plants: Yesterday, Today, and Tomorrow", Plant Physiology, 147(2008),456-468, http://dx.doi.org/10.1104/pp.108.117275.

[15] A.E.M. El-Misiery, E. Ahmed," On a fractional model for earthquakes", Appl. Comput. Math. 178 (2006) 207-211. http://dx.doi.org/10.1016/j.amc.2005.10.011.

[16] A.M.A. El-Sayed, I.L. El-Kalla, E.A.A. Ziada," Analytical and numerical solutions of multi-term nonlinear fractional orders differential equations", Applied Numerical Mathematics 60 (2010) 788 797, http://dx.doi.org/10.1016/j.apnum.2010.02.007.

[17] A.M.A. El-Sayed, M.E. Nasr," Existence of uniformly stable solutions of non-autonomous discontinuous dynamical systems", J.Egyptian Math. Soc. (2011) 19, 91-94. http://dx.doi.org/10.1016/j.joems.2011.09.006.

[18] A.M.A. El-Sayed," On the existence and stability of positive solution for a nonlinear fractional-order differential equation and some applications", Alex. J. Math. 1 (2010) 1-10.

[19] A.M.A. El-Sayed, S.Z. Rida, A.A.M. Arafa," Exact solutions of some time-fractional biological population models by using generalized differential transform method", Int. J. Math. Model. Simul. Appl. 3 (2010) 231-239.

[20] A.M.A. El-Sayed, S.Z. Rida, A.A.M. Arafa," On the Solutions of Time-fractional Bacterial Chemotaxis in a Diffusion Gradient Chamber", Int. J. Nonlinear Sci. Numer., 7(2009) 485-492.

[21] A.M.A. El-Sayed, A. E. M. El-Mesiry, and H. A. A. El-Saka,' Numerical solution for multi-term fractional (arbitrary) orders differential equations", Comput. Appl. Math., 23(2004)33-54. http://dx.doi.org/10.1590/S0101-82052004000100002.

[22] M.A.C. Groenenboom, P. Hogeweg," Modelling the dynamics of viral suppressors of RNA silencing”, J. R. Soc. Interface 9(2012), 436-447. http://dx.doi.org/10.1098/rsif.2011.0361.

[23] C.P. Li and F.R. Zhang," A survey on the stability of fractional differential equations", Eur. Phys. J. Special Topics 193 (2011) 27-47. http://dx.doi.org/10.1140/epjst/e2011-01379-1. 
[24] W. Lin," Global existence theory and chaos control of fractional differential equations", J. Math. Anal. Appl. 332 (2007) 709-726, http://dx.doi.org/10.1016/j.jmaa.2006.10.040.

[25] S. Mlotshwa, O. Voinnet, M. Florian Mette, M. Matzke, H. Vaucheret, S. Wei Ding, G. Pruss, V. B. Vance, "RNA Silencing and the Mobile Silencing Signal", The Plant Cell, (2002) 289301,http://dx. doi: 10.1105/tpc.001677.

[26] S. Nikolov," Dynamics and Complexity in a Time Delay Model of RNA Silencing with Periodic Forcing", Bioautomation, 10(2008) 112.

[27] Z. Odibat and N. Shawagfeh," Generalized Taylor's formula", Appl. Math. Comput. 186 (2007) 286-293, http://dx.doi.org/10.1155/2015/507970.

[28] Z. Odibat, and Shaher Moamni," An algorithm for the numerical solution of differential equations of fractional order", J. Appl. Math \& Informatics, 26(2008) $15-27$.

[29] L.M. Petrovic, D.T. Spasic, T.M. Atanackovic," On a mathematical model of a human root dentin", Dental Materials, 21(2005), 125128. http://dx.doi.org/10.1016/j.dental.2004.01.004.

[30] S. Pfeffer, G. Meister, M. Landthaler, T. Tuschl," RNA silencing", B.I.F. Futura, 20 (2005).

[31] F.A. Rihan," Numerical Modeling of Fractional-Order Biological Systems", Abstract and Applied Analysis, 2013, http://dx.doi.org/10.1155/2013/816803.

[32] V. Suat Ertürk, Zaid M. Odibat, Shaher Momani," An approximate solution of a fractional order differential equation model of human T-cell lymphotropic virus I (HTLV-I) infection of CD4+T-cells", Computers and Mathematics with Applications 62 (2011) 9961002. http://dx.doi.org/10.1016/j.camwa.2011.03.091

[33] What is RNA? : http://www.umassmed.edu/rti/biology/rna-faq/

[34] Why is RNA important to the cell? https://www.reference.com/science/rna-important-cellbcdd $937 \mathrm{bd} 3 \mathrm{cbc} 9 \mathrm{~b} 3$. 